\title{
ANÁLISE DA INFLUÊNCIA DA DENSIDADE DA MISTURA NA MODELAGEM DA EXPANSÃO VOLUMÉTRICA DE SOLVENTES ORGÂNICOS USANDO $\mathrm{CO}_{2}$ A ALTA PRESSÃO
}

\author{
S. G. CARDOSO ${ }^{1}$, M. L. P. M. $\operatorname{SILVA}^{1}$, G. M. N. $\operatorname{COSTA}^{1}, \underline{\text { S. A. B. VIEIRA DE MELO }}{ }^{1}$ \\ ${ }^{1}$ Universidade Federal da Bahia, Escola Politécnica, Programa de Engenharia Industrial \\ E-mail para contato: sabvm@ufba.br
}

\begin{abstract}
RESUMO - A análise da influência da densidade da mistura na modelagem da expansão volumétrica para quatro solventes orgânicos expandidos por $\mathrm{CO}_{2}$ a alta pressão é o foco desse trabalho. O comportamento da expansão volumétrica é observado através de termos calculados em função da densidade, para se determinarem quais são os pontos onde o erro da expansão vai ser maior ou menor, bem como se esse erro tem uma tendência crescente ou decrescente. Os resultados obtidos são de grande utilidade para o dimensionamento dos vasos de precipitação em processos que utilizam solventes orgânicos expandidos por $\mathrm{CO}_{2}$ denso.
\end{abstract}

\section{INTRODUÇÃO}

Em processos de precipitação de partículas usando solventes orgânicos expandidos por gases densos, tais como GAS, SAS e ARISE, é crucial determinar a priori o grau de expansão volumétrica da fase líquida (Vieira de Melo et al., 2013; Scurto et al., 2009). Alguns solventes orgânicos, quando expandidos por fluidos supercríticos, podem ter um aumento de mais de dez vezes o volume inicial, o que deve ser conhecido para o correto dimensionamento dos vasos de precipitação. Por exemplo, a expansão volumétrica da acetona pelo $\mathrm{CO}_{2}$, a $40{ }^{\circ} \mathrm{C}$ e 80 bar, é da ordem de 1000\% (Denardin et al., 2013).

Espera-se que uma equação de estado cúbica bem ajustada seja capaz de prever com precisão o grau de expansão volumétrica de solventes orgânicos por gases densos. Na prática, entretanto, é muito mais fácil, rápida e barata a determinação experimental da expansão volumétrica de solventes orgânicos por gases densos do que a determinação experimental da densidade da mistura líquida (Denardin et al., 2013; Su, 2012; Kordikowski et al., 1995). Além disso, encontra-se na literatura um bom número de dados experimentais de pressão e composição no ponto de bolha para sistemas binários do tipo solvente orgânico e gás denso, sobretudo para o dióxido de carbono, sem que haja os correspondentes dados experimentais de densidade da fase líquida (Bezanehtak et al., 2002; Day et al., 1996; Lazzaroni et al., 2005). Por essa razão, é comum ajustar as equações de estado cúbicas usando apenas dados de pressão e composição nos pontos de bolha e assumir que o modelo será capaz de prever com precisão a densidade da mistura líquida. Contudo, estudos na literatura relatam que nem sempre essa consideração pode ser feita, podendo ocorrer grandes erros na predição da densidade, o que por consequência levaria ao cálculo equivocado do grau de expansão volumétrica (de la Fuente Badilla et al., 2000). 
Por outro lado, observa-se ainda na literatura que o grau de expansão volumétrica, calculado a partir de uma equação de estado cúbica ajustada apenas com dados de pressão e composição dos pontos de bolha, muitas vezes consegue reproduzir com boa precisão dados experimentais de expansão volumétrica obtidos nas mesmas condições, mesmo quando o modelo não é capaz de calcular com precisão a respectiva densidade da mistura líquida (Costa et al., 2012). Como a expansão volumétrica e a densidade da fase líquida, por definição, estão diretamente relacionadas, surgiu a seguinte questão: porque modelos que não conseguem descrever com precisão a densidade da mistura líquida são capazes de fazer uma boa predição do grau de expansão volumétrica?

O objetivo do presente trabalho é tentar responder a essa pergunta, analisando a influência da densidade da mistura na modelagem da expansão volumétrica de solventes orgânicos usando $\mathrm{CO}_{2}$ a alta pressão.

\section{MODELAGEM TERMODINÂMICA}

A expansão volumétrica relativa da fase líquida, por definição, é a diferença entre o volume total final da fase líquida e o volume total inicial da fase líquida dividida pelo volume total inicial da fase líquida, lembrando que o volume total inicial da fase líquida é igual ao volume total do solvente puro. Tomando como base $1 \mathrm{~mol}$ de solvente (de la Fuente et al., 2000), a expansão volumétrica da fase liquida em que o $\mathrm{CO}_{2}$ denso atua se dissolve é calculada por:

$$
\frac{\Delta V}{V}=\frac{\rho_{2}\left(T, P_{0}\right)}{\rho_{L}\left(T, P, x_{1}\right)} \cdot\left[\frac{x_{1}}{\left(\left(1-x_{1}\right)\right.} \cdot \frac{M_{1}}{M_{2}}+1\right]-1
$$

onde $\Delta V / V$ indica a expansão volumétrica relativa do solvente com a adição de $\mathrm{CO}_{2}, x_{1}$ é a fração molar do $\mathrm{CO}_{2}$ na solução, $\rho_{L}$ e $\rho_{2}$ são a densidade da mistura e do solvente puro, respectivamente, $M_{1}$ e $M_{2}$ os pesos moleculares do $\mathrm{CO}_{2}$ e do solvente, $P$ e $T$ a pressão e a temperatura na qual se realiza a expansão, e $P_{0}$ a pressão atmosférica.

Para melhor avaliação da influência dos vários termos da Eq. (1) no cálculo da expansão volumétrica relativa, foram considerados dois termos:

$$
\begin{aligned}
& A=\left[\frac{x_{1}}{\left(\left(1-x_{1}\right)\right.} \cdot \frac{M_{1}}{M_{2}}+1\right] \\
& B=\frac{\rho_{2}\left(T, P_{0}\right)}{\rho_{L}\left(T, P, x_{1}\right)} \cdot\left[\frac{x_{1}}{\left(\left(1-x_{1}\right)\right.} \cdot \frac{M_{1}}{M_{2}}+1\right]
\end{aligned}
$$


Para o cálculo da densidade da mistura líquida e do solvente puro foi usada a equação de estado de Peng-Robinson, empregando as regras de mistura quadráticas clássicas, com dois parâmetros de interação binárias ajustáveis, $k_{i j}$ e $l_{i j}$ (Poling et al., 2001).

Para melhorar a qualidade do cálculo da densidade e manter a qualidade da descrição do equilíbrio, foi utilizada a correção de Peneloux (Pedersen et al., 2012), expressa por:

$$
\mathrm{V}_{\mathrm{pen}}=\mathrm{V}_{\mathrm{pr}}-\mathrm{c}
$$

onde $V_{p r}$ é o volume molar calculado pela equação de Peng-Robinson, $c$ é o fator de correção de Peneloux e $V_{p e n}$ é o volume molar corrigido. O cálculo do fator $c$ para o componente puro é feito por:

$$
c=\frac{0,50033 \cdot R \cdot T_{c}}{P_{c}} \cdot[0,25969-(0,29056-0,08775 \cdot w)]
$$

sendo $T_{c}$ e $P_{c}$ a temperatura crítica e a pressão crítica, respectivamente, e $w$ o fator acêntrico.

A regra de mistura para a correção de Peneloux é expressa como:

$c=\sum_{i} x_{i} \cdot c_{i}$

Para a estimação dos parâmetros de interação binária $k_{i j}$ e $l_{i j}$ foram utilizados dados experimentais de pressão de bolha e a seguinte função objetivo (FO), otimizada via método SIMPLEX:

$$
F O=\min \sum_{i}\left|\frac{P_{\text {exp }}-P_{\text {calc }}}{P_{\text {exp }}}\right|
$$

\section{RESULTADOS E DISCUSSÃO}

As informações relevantes sobre os dados experimentais de equilíbrio líquido-vapor utilizados na estimação dos parâmetros de interação binária são apresentadas na Tabela 1 .

Tabela 1 - Dados experimentais de equilíbrio liquido-vapor para sistemas binários.

\begin{tabular}{lcccl}
\hline Sistemas binários & $\mathrm{T}(\mathrm{K})$ & $\mathrm{P}(\mathrm{atm})$ & $\begin{array}{c}\mathrm{N}^{\mathrm{o}} \text {. de } \\
\text { pontos }\end{array}$ & \multicolumn{1}{c}{ Referência } \\
\hline $\mathrm{CO}_{2}$-metanol & 308,15 & $15,2-71,7$ & 7 & Bezanehtak et al. (2002) \\
$\mathrm{CO}_{2}$-etanol & 313,15 & $25,5-77,6$ & 14 & Sih et al. (2008) \\
$\mathrm{CO}_{2}$-acetona & 313,15 & $7,7-63,5$ & 9 & Day et al. (1996) \\
$\mathrm{CO}_{2}$-DMSO & 298,15 & $4,1-60,1$ & 16 & Kordikowski et al. (1995) \\
\hline
\end{tabular}


Como pode ser observado na Eq. (1), a qualidade da descrição da expansão volumétrica está diretamente relacionada à qualidade da descrição da densidade. Por esta razão, foi realizado inicialmente um estudo da influência da correção de Peneloux na densidade, e os resultados estão apresentados na Tabela 2. Pelo exposto, observou-se que o uso dessa correção é altamente recomendável.

Tabela 2 - Erro percentual no cálculo da densidade: influência da correção de Peneloux.

\begin{tabular}{lcc}
\hline & $\Delta \rho(\%)$ sem correção de Peneloux & $\Delta \rho(\%)$ com correção de Peneloux \\
\hline $\mathrm{CO}_{2}$-metanol & 18,2 & 5,3 \\
$\mathrm{CO}_{2}$-etanol & 15,6 & 9,4 \\
$\mathrm{CO}_{2}$-acetona & 28,1 & 7,5 \\
$\mathrm{CO}_{2}$-DMSO & 26,8 & 9,7 \\
\hline
\end{tabular}

A comparação entre os valores calculados e os valores experimentais para a expansão volumétrica e a densidade em função das frações molares de $\mathrm{CO}_{2}$ adicionados, para os sistemas $\mathrm{CO}_{2}$-metanol e $\mathrm{CO}_{2}$-etanol, é apresentada nas Figuras 1 e 2, nas quais podem-se observar que, para qualquer fração molar de $\mathrm{CO}_{2}$, os valores calculados de expansão volumétrica são sempre inferiores aos valores experimentais. Dentre os sistemas analisados estes resultados demonstram que os menores erros da expansão volumétrica são para o sistema $\mathrm{CO}_{2}$-DMSO (24\%), mesmo o erro da densidade não sendo o menor (9.7\%). A análise da densidade, para os sistemas $\mathrm{CO}_{2}$-etanol e $\mathrm{CO}_{2}$-acetona, apresenta valores calculados sempre superiores aos valores experimentais. Para o sistema $\mathrm{CO}_{2}$-metanol, quando a fração molar do $\mathrm{CO}_{2}$ é menor do que 0,4 , o valor experimental é maior do que o calculado, enquanto para fração molar maior do que 0,4 , este comportamento é o inverso. Para o sistema $\mathrm{CO}_{2^{-}}$ DMSO, em qualquer composição, o valor experimental é sempre maior do que o valor calculado. Tendo em vista os resultados apresentados nas Figuras 1 e 2, torna-se absolutamente relevante a análise dos termos expressos pelas Eqs. (2) e (3) para melhor compreensão da influência da densidade na expansão volumétrica da mistura. Outro fato importante diz respeito às escalas empregadas no cálculo da expansão volumétrica e da densidade. Apesar dos valores próximos ou distantes de uma ou outra escala, uma avaliação criteriosa necessita do cálculo do erro percentual, o que é apresentado a seguir.
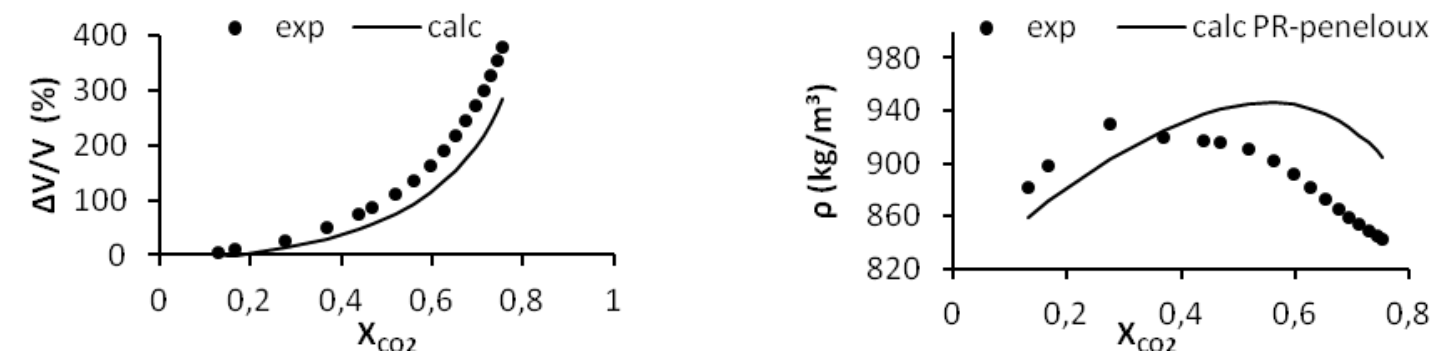

Figura 1 - Comparação entre valores calculados e experimentais da expansão volumétrica e da densidade versus fração molar de $\mathrm{CO}_{2}$, usando a correção de Peneloux, para o sistema $\mathrm{CO}_{2-}$ metanol a $308,15 \mathrm{~K}$. 

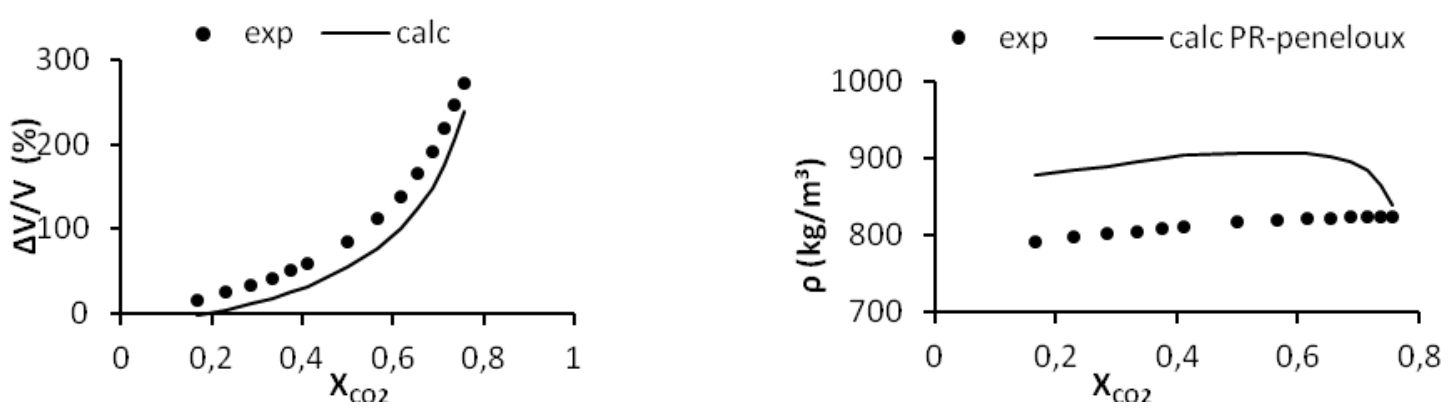

Figura 2 - Comparação entre valores calculados e experimentais da expansão volumétrica e da densidade versus fração molar de $\mathrm{CO}_{2}$, usando a correção de Peneloux, para o sistema $\mathrm{CO}_{2}$ etanol a $313,15 \mathrm{~K}$.

Para o sistema $\mathrm{CO}_{2}$-metanol, os resultados das análises separadas de cada fator $\mathrm{A}$ e $\mathrm{B}$, calculados segundo as Eqs. (2) e (3), em função da fração molar de $\mathrm{CO}_{2}$, são exibidos na Fig. 3. O mesmo tipo de análise foi realizado para os outros sistemas e observou-se o mesmo padrão de comportamento. Segundo a Eq. (2), o parâmetro A é adimensional e função somente da fração molar e dos pesos moleculares, e tem comportamento único como mostrado na Fig. 3. Nesta figura é importante observar que os parâmetros "A" e "B" possuem a mesma ordem de grandeza.
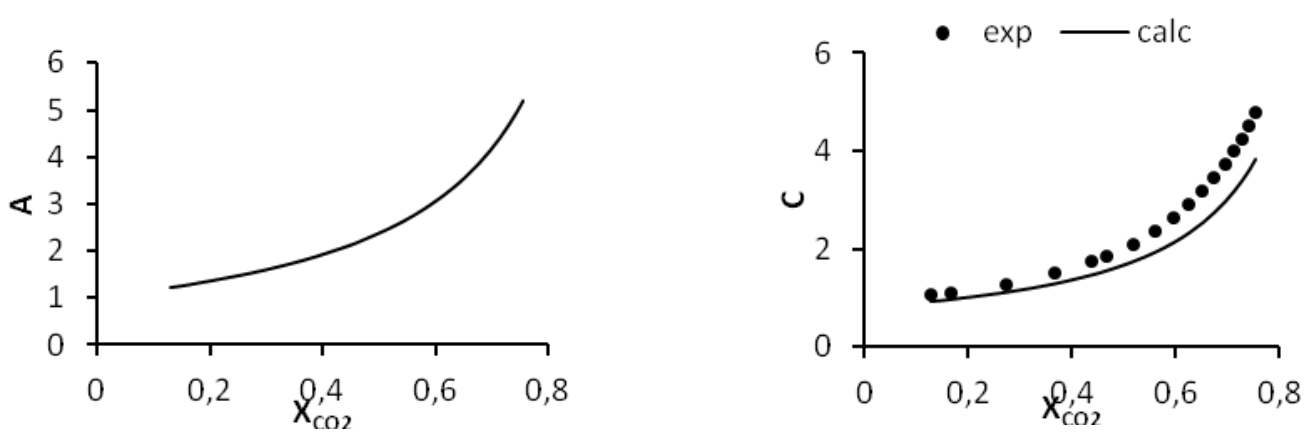

Figura 3 - Parâmetros A e B versus fração molar de $\mathrm{CO}_{2}$ para o sistema $\mathrm{CO}_{2}$-metanol a $308,15 \mathrm{~K}$.

Após esta análise, foi verificado ser também importante, para uma avaliação melhor do erro percentual, reescrever a Eq. (1) em função somente da Eq. (3), resultando em:

$$
\frac{\Delta V}{V}=B-1
$$

Para avaliar o comportamento da expansão volumétrica, é necessário avaliar a influência do parâmetro "B" e, consequentemente, da densidade, no seu cálculo. Esta avaliação pode ser monitorada por diferentes tipos de erros percentuais, podendo ser expressos pela Eqs. (9) e (10).

$$
\operatorname{ErroV}(\%)=\left[\frac{(\Delta V / V)_{\exp }-(\Delta V / V)_{\text {calc }}}{(\Delta V / V)_{\exp }}\right] \cdot 100 \%=\frac{B_{\exp }-B_{\text {calc }}}{B_{\exp }-1} \cdot 100 \%
$$




$$
\operatorname{ErroB}(\%)=\frac{B_{\text {exp }}-B_{\text {calc }}}{B_{\text {exp }}} \cdot 100 \%
$$

Neste caso, deve-se analisar o comportamento dos termos $\left(\mathrm{B}_{\exp }-\mathrm{B}_{\text {calc }}\right)$ e $\left(\mathrm{B}_{\exp }-1\right)$. Se apenas o termo $\left(\mathrm{B}_{\exp }-\mathrm{B}_{\text {calc }}\right)$ aumentar, não implica que o erro irá aumentar, assim como se o termo $\left(\mathrm{B}_{\exp }-1\right)$ aumentar não implica que o $\operatorname{ErroV}(\%)$ irá diminuir. Caso as variações desses dois termos sejam próximas, mesmo que o valor seja sempre crescente, pode ocorrer uma compensação e o erro da expansão vai se manter constante. Logo, constata-se que, para diminuição no erro da expansão volumétrica, o valor da variação de $\left(\mathrm{B}_{\exp }-1\right)$ deve ser maior do que a do termo $\left(\mathrm{B}_{\exp }-\mathrm{B}_{\text {calc }}\right)$. Considerem-se dois valores consecutivos para fração molar de $\mathrm{CO}_{2}$. Avaliando-se a diferença entre os valores do parâmetro "B" experimental e calculado para dois valores de fração molar de $\mathrm{CO}_{2}$, pode-se escrever:

$$
\begin{aligned}
& \operatorname{VARB} 1(\%)=\left[\frac{\left(B_{\text {exp }}-B_{\text {calc }}\right)_{i+1}-\left(B_{\text {exp }}-B_{\text {calc }}\right)_{i}}{\left(B_{\text {exp }}-B_{\text {calc }}\right)_{i}}\right] \cdot 100 \% \\
& \operatorname{VARB} 2(\%)=\left[\frac{\left(B_{\text {exp }}-1\right)_{i+1}-\left(B_{\text {exp }}-1\right)_{i}}{\left(B_{\text {exp }}-1\right)_{i}}\right] \cdot 100 \% \\
& \operatorname{VARB3}(\%)=\left[\frac{\left(B_{\text {exp }}\right)_{i+1}-\left(B_{\text {exp }}\right)_{i}}{\left(B_{\exp }\right)_{i}}\right] \cdot 100 \%
\end{aligned}
$$

Os erros calculados pelas Eqs. (11) a (13) em função da fração molar de $\mathrm{CO}_{2}$, para os sistemas $\mathrm{CO}_{2}$-metanol, $\mathrm{CO}_{2}$-etanol, $\mathrm{CO}_{2}$-acetona e $\mathrm{CO}_{2}$-DMSO, são ilustrados na Figura 6.

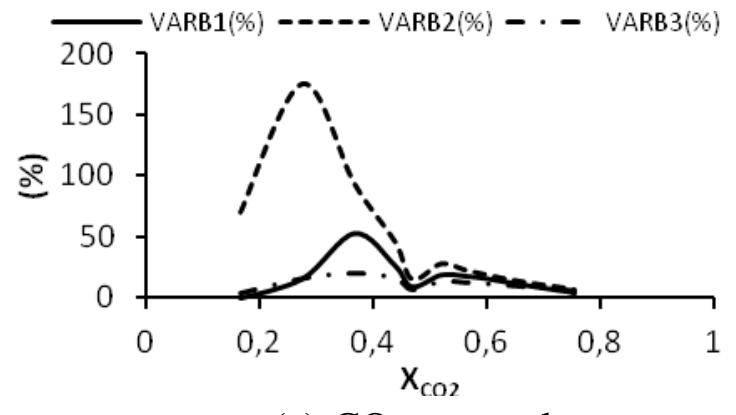

(a) $\mathrm{CO}_{2}$-metanol



(c) $\mathrm{CO}_{2}$-acetona

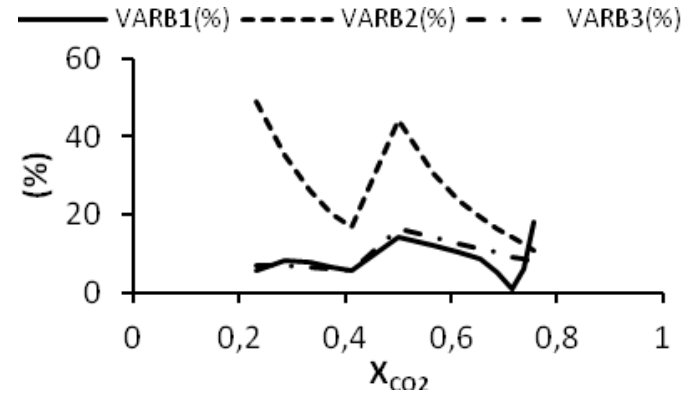

(b) $\mathrm{CO}_{2}$-etanol



(d) $\mathrm{CO}_{2}$-DMSO

Figura 4 - Erros para os sistemas $\mathrm{CO}_{2}$-metanol, $\mathrm{CO}_{2}$-etanol, $\mathrm{CO}_{2}$-acetona e $\mathrm{CO}_{2}$-DMSO. 
De acordo com a Eq. (12), o erro da expansão volumétrica está relacionado com os termos $\left(\mathrm{B}_{\exp }-\mathrm{B}_{\text {calc }}\right)$ e $\left(\mathrm{B}_{\exp }-1\right)$. Estes termos são funções da densidade da mistura líquida, logo o comportamento da densidade influencia o comportamento da expansão volumétrica. Com a ajuda da Fig. 4, pode-se prever se o erro da expansão volumétrica vai diminuir ou aumentar, bem como se o erro do parâmetro " $B$ " vai diminuir ou aumentar, fazendo-se apenas a análise da variação dos termos $\left(B_{\exp }-B_{\text {calc }}\right),\left(B_{\exp }-1\right)$ e $B_{\exp }$ através das Eqs. (11), (12) e (13), respectivamente.

Para se entender como o erro da expansão volumétrica aumenta com o aumento da fração molar de $\mathrm{CO}_{2}$, é importante observar os gráficos dos termos $\left(\mathrm{B}_{\exp }-\mathrm{B}_{\text {calc }}\right)$ e $\left(\mathrm{B}_{\exp }-1\right)$ em função da fração molar de $\mathrm{CO}_{2}$. Analisando-se a Figura 5, percebe-se que a diferença entre o termo $\left(\mathrm{B}_{\exp }-\mathrm{B}_{\text {calc }}\right)$ e o termo $\left(\mathrm{B}_{\exp }-1\right)$ diminui com a diminuição da fração molar de $\mathrm{CO}_{2}$.

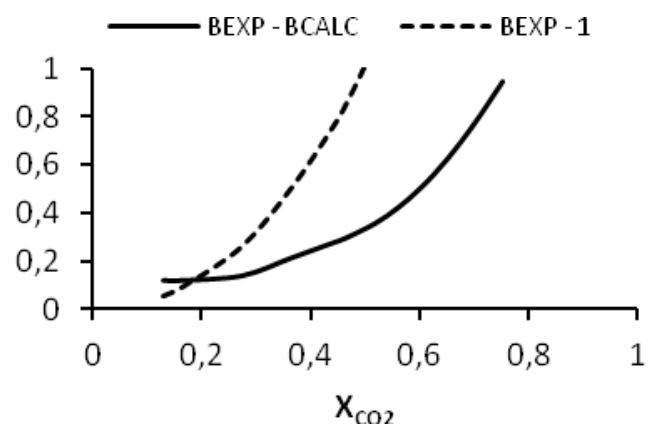

(a) $\mathrm{CO}_{2}$-metanol

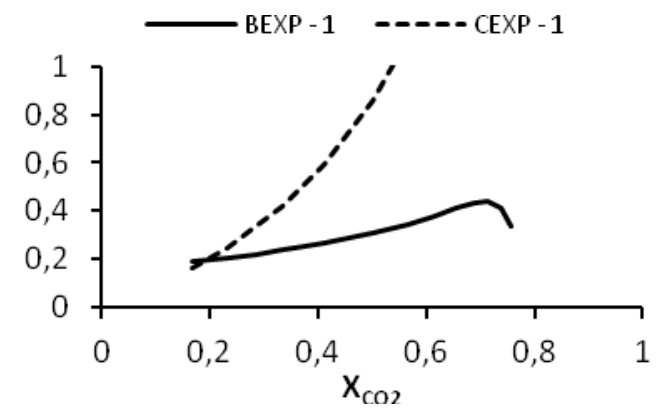

(c) $\mathrm{CO}_{2}$-acetona

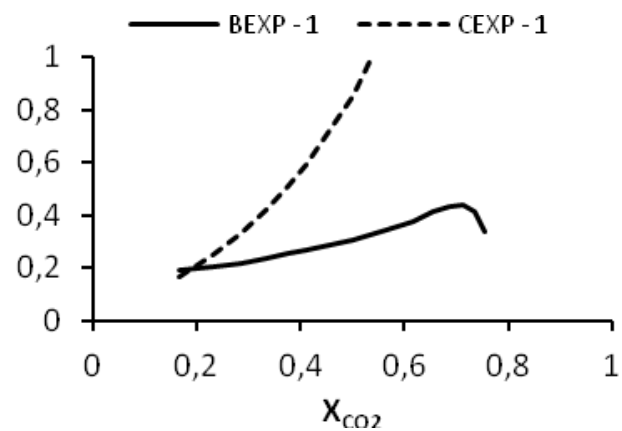

(b) $\mathrm{CO}_{2}$-etanol

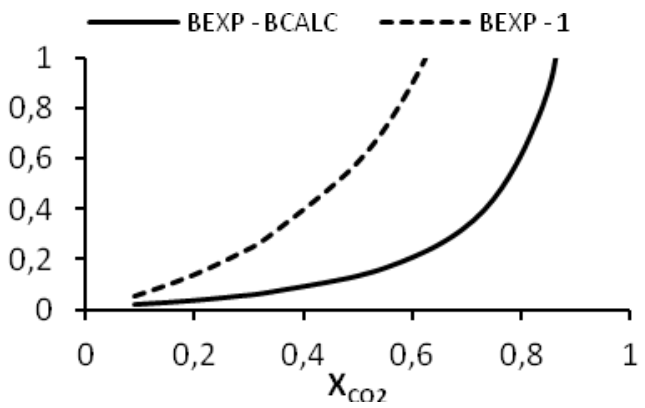

(d) $\mathrm{CO}_{2}$-DMSO

Figura 5 - $\left(\mathrm{B}_{\exp }-\mathrm{B}_{\exp }\right)$ e $\left(\mathrm{B}_{\exp }-1\right)$ em função da fração molar de $\mathrm{CO}_{2}$ para os sistemas $\mathrm{CO}_{2^{-}}$ metanol, $\mathrm{CO}_{2}$-etanol, $\mathrm{CO}_{2}$-acetona e $\mathrm{CO}_{2}$-DMSO.

\section{CONCLUSÃO}

Estudou-se a influência da densidade da mistura líquida no cálculo da expansão volumétrica de quatro solventes orgânicos expandidos por $\mathrm{CO}_{2}$ a alta pressão. Para entender o comportamento da expansão volumétrica foi necessário fazer a análise dos termos $\left(\mathrm{B}_{\exp }-\right.$ $\left.\mathrm{B}_{\text {calc }}\right)$ e $\left(\mathrm{B}_{\exp }-1\right)$, calculados em função da densidade. Contudo, não necessariamente o sistema com menor erro na predição da densidade apresenta a melhor predição da expansão volumétrica. Observando-se o comportamento da expansão volumétrica em função dos termos $\left(B_{\exp }-B_{\text {calc }}\right)$ e $\left(B_{\exp }-1\right)$, pode-se determinar onde o erro da expansão é maior ou 
menor, bem como se sua tendência é crescente ou decrescente. Constatou-se ainda a importância da correção do volume molar da mistura utilizando a expressão de Peneloux.

\section{REFERÊNCIAS}

BEZANEHTAK, K.; COMBES, G. B.; DEHGHANI, F.; FOSTER, N. R.; TOMASKO D. L. Vapor-liquid Equilibrium for Binary Systems of Carbon Dioxide + Methanol, Hydrogen + Methanol, and Hydrogen + Carbon Dioxide at High Pressures, J. Chem. Eng. Data, v. 47, p. 161-168, 2002.

COSTA, G.M.N.; MATOS, R.L.; PEREIRA, V.J.; SANTANA, G.L.; VIEIRA DE MELO, S.A.B. Modeling High Pressure Phase Behavior of the Binary System Solvent/Antisolvent for SAS Processing of Pharmaceuticals Using PR-LCVM-UNIFAC Equation of State, Proc. $10^{\text {th }}$ International Symposium on Supercritical Fluids, May, 13-16, San Francisco, USA, 2012.

DAY, C.; CHANG, C. J.; CHEN, C. Phase Equilibrium of Ethanol $+\mathrm{CO}_{2}$ and Acetone $+\mathrm{CO}_{2}$ at Elevated Pressures, J. Chem. Eng. Data, v. 41, p. 839-843, 1996.

DENARDIN, F.G.; VIEIRA DE MELO, S.A.B.; MAMMUCARI, R.; FOSTER, N.R. Phase transition and volume expansion in $\mathrm{CO}_{2}$-expanded liquid systems, Chemical Engineering Transactions, v. 32, p. 529-534, 2013.

DE LA FUENTE BADILLA, J.C.; PETERS, C.J.; DE SWAAN ARONS, J. Volume expansion in relation to the gas-antisolvent process, J. Supercritical Fluids, v.17, p. 13-23, 2000.

KORDIKOWSKI, A.; SCHENK, A.P.; VAN NIELEN, R.M.; PETERS C.J. Volume expansions and vapor-liquid equilibria of binary mixtures of a variety of polar solvents and certain near-critical solvents, J. Supercritical Fluids, v.8, p. 205-216, 1995.

LAZZARONI, M.J.; BUSH, D.; BROWN, J.S.; ECKERT, C.A. High-pressure vaporliquid equilbria of some carbon dioxide + organic binary systems, J. Chem. Eng. Data, v. 50, p. 60-65, 2005.

PEDERSEN, K. S.; CHRISTENSEN, P. L.; AZEEM, S. J. Phase Behavior of Petroleum Reservoir Fluids. New York: Taylor \& Francis Group, 2012.

POLING, B. E.; PRAUSNITZ, J. M.; O'CONNELL, J. P. The properties of gases and liquids, $5^{\text {th }}$. ed. New York: McGraw-Hill, 2001.

SCURTO, A.M.; HUTCHENSON, K.; SUBRAMANIAM, B. Gas-Expanded Liquids: Fundamentals and Applications, In Gas-Expanded Liquids and Near-Critical Media; Hutchenson, K., et al., ACS Symposium Series, American Chemical Society: Washington, DC, p. 3-37, 2009.

SIH, R.; ARMENTI, M.; MAMMUCARI, R.; DEHGHANI, F.; FOSTER, N.R. Viscosity measurements on saturated gas-expanded liquid systems-Ethanol and carbon dioxide, J. Supercritical Fluids, v. 43, p. 460-468, 2008.

SU, C.-S. Prediction of volumetric properties of carbon dioxide-expanded organic solvents using the Predictive Soave-Redlich-Kwong (PSRK) equation of state, $J$. Supercritical Fluids, v. 72, p. 223-231, 2012.

VIEIRA DE MELO, S.A.B.; DANH, L.T.; MAMMUCARI, R.; FOSTER, N.R. Dense $\mathrm{CO}_{2}$ precipitation of levothyroxine sodium by GAS and ARISE processes: A comparative study on morphology and particle size distributions, J. Supercritical Fluids (in press), 2013. 